\title{
O que devemos ter em conta para definir corretamente os termos distance learning, e-learning e m-learning?
}

\section{What should we consider to correctly define the terms distance learning, e-learning, and m-learning?}

\author{
João Paulo Vagarinho*
}

\begin{abstract}
RESUMO
A investigação agregada ao ensino a distância encontra dificuldades quando se pretende comparar estudos sobre definições, visto que, amplas, estão associadas a diferentes contextos e interesses de alguns autores, muitas vezes incompletas e com falta de rigor. Portanto, essas definições devem ser clarificadas, ordenadas e definidas corretamente. Analisei mais de 60 artigos que envolvem os três conceitos e encontrei uma forma para ter em conta ao construi-los corretamente. Identifiquei um conjunto de características e subcaracterísticas que recomendo a todos os interessados para a construção correta das definições distance learning, e-learning e m-learning.
\end{abstract}

Palavras-chave: Ensino a distância. Ensino eletrónico. Ensino móvel.

\begin{abstract}
Research, as related to distance learning, encounters difficulties when comparing studies of definitions, insofar as they are broad, are associated with different contexts and interests of some authors, and often incomplete and lacking in rigor. These definitions must be clarified, ordered and defined correctly. I have reviewed more than sixty articles that involve the three concepts and found a way to consider building them correctly. I have identified a set of characteristics and sub-characteristics that I recommend
\end{abstract}

"Universidade de Vigo. Vigo, Pontevedra, Espanha. E-mail: joao.paulo.vagarinho@gmail. com. https://orcid.org/0000-0003-0084-7810 
all interested parties to consider for the correct construction of the definitions of distance learning, e-learning, and m-learning.

Keywords: Distance learning. E-learning. M-learning.

\section{Introdução}

A educação a distância iniciou-se há quase dois séculos (Saba, 2016, pp. 21-30), neste período de tempo várias gerações possuíram os seus modelos, por exemplo, o modelo por correspondência com base na impressão, o modelo de fita de áudio e de fita de vídeo, o modelo via rádio e TV (Ding, Niu, \& Han, 2010, pp. 582-592). As tecnologias de informação e as tecnologias educacionais evoluem constantemente (Bottino, 2004, pp. 553-567; Pahl, 2013, pp. 99-114) e com a internet, a globalização e o objetivo de atingir uma sociedade do conhecimento, a aprendizagem, o ensino e a investigação mudou e continua em constante mudança (Kerr, 2014, pp. 156-160). Perante este cenário, as instituições de educação têm realizado profundas e rápidas alterações na melhoria do ensino para os seus alunos (Hürsen, Özçınar, Özdamlı, \& Uzunboylu, 2011, pp. 59-66), principalmente, recorrendo à utilização de várias tecnologias para oferecer formação através de várias formas de entrega (Motiwalla, 2007, pp. 581-596). Para além do distance learning e em conjunto com essas novas formas de entrega de ensino, surgiram igualmente novos termos agregados, nomeadamente, o e-learning ((Selim, 2007, pp. 396-413) e o m-learning (Cavus \& Ibrahim, 2009, pp. 78-91), como consequência as partes interessadas nesta área ainda não conseguiram encontrar consensos para algumas definições (Anohina, 2005, pp. 91-102; Grossnickle, 2016, pp. 23-60). Devido a esta falta de rigor, em particular nas formas de entrega da educação a distância, é difícil identificar as diferenças significativas de cada definição (Keegan, 1996, p. 224; VanDerLinden, 2014, pp. 75-85), como consequência é difícil para os pesquisadores realizarem novos estudos porque as conclusões entram em conflito com as definições. Uma forma para ultrapassar este problema é encontrar um conjunto de características e subcaracterísticas agregado a cada definição, que permitirá aos interessados, construir definições mais rigorosas, corretas e adequadas. Característica significa que cada definição possui uma ou várias propriedades que são particulares, capaz de se identificar, definir ou classificar de acordo com as suas qualidades ou debilidades (Ramírez, Rogan, Valdivia, Varas, \& Kiwi, 2016, pp. 977-989; Brito, 2012, pp. 580-593). 


\section{Revisão da literatura}

\section{Distance learning}

O ensino a distância é um termo que muitas vezes é utilizado por vários autores com significados diferentes (Anohina, 2005, pp. 91-102) e muitas vezes o termo ensino a distância é confundido com o termo educação a distância (Cropley \& Kahl, 1983; Joksimović, Gašević, Loughin, Kovanović, \& Hatala, 2015, pp. 204-217). De acordo com King, Young, Drivere-Richmond, \& Schrader (2001, pp. 1-14), ensino a distância é referenciado como melhoramento da capacidade na aquisição de conhecimento enquanto educação a distância é composta por ações para ensinar e aprender. Na história sobre o ensino a distância, encontramos autores a salientar que, inicialmente, os cursos de ensino a distância eram feitos por meio de correspondência recorrendo ao papel ((Banas \& Emory, 1998, pp. 365-383), posteriormente, esse meio de entrega evoluiu para o rádio (Passerini \& Granger, 2000, pp. 1-15), transmitindo em rádio frequência (Dede, 1990, pp. 247-264). Com a introdução da tecnologia, os cursos universitários e cursos profissionais eram produzidos em vídeos gravados em fitas, contendo som e imagem para serem utilizados na TV, esses conteúdos eram distribuídos via correio ou fax (Katz, 2002, pp. 2-9). Esses autores focam apenas nas suas definições o componente de acesso, modo de entrega e os cursos oferecidos. Outros autores destacam que, no ensino a distância, a interação entre o alunoprofessor, aluno-aluno e professor-aluno não existe (Chickering \& Ehrmann, 1996, pp. 3-7), ou que existe de forma remota (Galusha, 1997, pp. 6-14), ou que a única forma de comunicar é enviando mensagens em papel (Allen et al., 2004, pp. 402-420). Poucos autores escrevem sobre acessibilidade e adaptabilidade (em termos de sistema) como características da definição, provavelmente porque não existe neste tipo de ensino, não só em termos de materiais de aprendizagem como também ao nível do suporte e admissão nos cursos (Mapuranga \& Nyenya, 2014, pp. 1-12; Rauner \& Maclean, 2008, p. 832).

\section{E-learning}

Um termo que evoluiu com base no ensino a distância foi o e-learning (Du, Liu, \& Wei, 2010). Dos vários artigos consultados podemos constatar que a definição de e-learning surge ligada à vantagem de possuir as mais recentes ferramentas tecnológicas para a educação (Tarus, Gichoya, \& Muumbo, 2015, pp. 120-140), a uma nova geração de sociedade baseada no conhecimento (Jones \& Lau, 2009, pp. 40-48), à reestruturação da oferta educativa e à adaptação dos ambientes de e-learning ao mundo académico atual (Kim \& Santiago, 2005, 
pp. 108-114), à promoção da mobilidade dos estudantes (Wachter, 2004, pp. 265-273), a uma maior afluência à aprendizagem, sobretudo na aprendizagem ao longo da vida (Liu G., 2010, pp. 218-222) ao crescimento económico de um país (Schweizer, 2004, pp. 674-692) ou ser considerado uma rede académica de conteúdos e um conjunto de processos que interagem entre estudantes, informação e promoção de aprendizagem (Tuul, Banzragch \& Saizmaa, 2016, pp. 181-193). Outros autores referem que a definição de e-learning está associada à disponibilização da aprendizagem apoiada por ferramentas eletrônicas digitais e media (Milrad, 2003, pp.151-164), suportados pelo desktop e laptop (Koper, 2005, pp. 3-20) e pelas tecnologias de internet e/ou intranet (Isaiah \& Martin, 2015, pp. 64-79), que permitem aceder a uma grande quantidade de recursos de informação e conhecimento (Hartshorne \& Ajjan, 2009, pp. 183198). Outros, porém, preferem abordar as interações entre alunos e professores (Sun, Tsai, Finger, Chen, \& Yeh, 2008, pp. 1183-1202), a aprendizagem colaborativa (Lyashenko \& Frolova, 2014, pp. 495-513), as atividades para os alunos realizarem - que podem ser online ou offline (Korucu \& Alkan, 2011, pp. 1925-1930) -, o modo síncrono (Boulos, Taylor, \& Breton, 2005, pp. 583593), a sua atração (Guri-Rosenblit, 2005, pp. 467-493), especialmente, quando ligado a ferramentas sociais como o Second Life que motivam os alunos na aprendizagem(Warburton, 2009, pp. 414-426). Outros salientam que a definição de e-learning está ligada às vantagens dos alunos poderem estudar a qualquer hora e em qualquer lugar (Du, Liu \& Wei, 2010, pp. 659-661), logo é conveniente em termos de flexibilidade de aprendizagem (Li, Duan, Fu \& Alford, 2012, pp. 933-948), sendo útil para fornecer educação em zonas rurais (Brown, 2005, pp. 299-315) ou para estudantes com dificuldade na deslocação a um espaço físico (Woodfine, Nunes, \& Wright, 2008, pp. 703-717), a grande oferta formativa que disponibiliza quer em cursos superiores de Licenciatura, Master ou PhD (McGill \& Klobas, 2009, pp. 496-508), quer em cursos MBA (Raaij \& Schepers, 2008, pp. 838-852), MOOC ou outro tipo de formação (Liyanagunawardena, Lundqvist \& Williams, 2015, pp. 557-569), a adaptabilidade dos sistemas tradicionais e a sua evolução para o aparecimento dos adaptive e-learning systems, os quais permitem vários tipos de técnicas para a aprendizagem (Alshammari, Anane, \& Hendley, 2014, pp. 79-85), tendo em consideração os aspetos cognitivos relativamente a materiais de aprendizagem, interações e outros conteúdos para alunos "sem e com deficiência" (Li J., 2010, pp. 2017-2020; Ozkan \& Koseler, 2009, pp. 1285-1296). Em todos os artigos mencionados, parece óbvio a falta de clareza quanto ao que são exatamente as características que podem acompanhar a definição de e-learning embora na perspetiva de proporcionar uma oportunidade de aprendizagem o consenso seja comum. 


\section{M-learning}

$\mathrm{O}$ termo m-learning, atualmente muito utilizado, surge nas definições ligado às tecnologias móveis para a educação, como um canal educativo alternativo, onde os alunos podem agora adquirir conhecimento em qualquer lugar (Popović, Marković, \& Popović, 2016, pp. 412-420), de acordo com o interesse, padrões de trabalho e oportunidade (Peters, 2007, pp. 1-16). Alguns autores preferem destacar na definição que o m-learning utiliza várias fontes de informação para inovar a aprendizagem e recorre aos minicomputadores de bolso (que são facilmente transportados) em conjunto com as redes sem fio ( $\mathrm{Ng} \&$ Nicholas, 2013, pp. 695-715). Na definição que outros autores apresentam, podemos também encontrar que a comunicação e a transferência de dados criados por tecnologias fixas podem reduzir significativamente a produtividade do trabalho e estudo (Sarrab, Shibli, \& Badursha, 2016, pp. 331-349) fora da sala de aula (Rosenberg, 2002, pp. 66-117), pelo que o ideal seria estar ligado a qualquer momento que seja desejado (Park Y., 2011), para tal, utiliza tecnologias inovadoras oferecidas pelos dispositivos móveis como iPod, iPhone, iPad (Ciampa, 2013, pp. 82-96), PDA's (Uzunboylu \& Ozdamli, 2011, pp. 544-556), laptops, MP3 e tablets (Tabuencaa, Kalzb, Drachslerb, \& Specht, 2015, pp. 53-74). O iPod, por exemplo, é utilizado como ferramenta de ensino que permite a utilização do Podcasting (Heilesen, 2010, pp. 1063-1068), isto é, o dispositivo é usado para ouvir ou assistir a uma transmissão de áudio ou vídeo, essas publicações estão na internet e o aluno pode assistir a elas onde quiser, quando quiser e como quiser (Evans, 2008, pp. 491-498). Para esses autores, os alunos conseguem desta forma ter acesso com facilidade à informação, à aprendizagem, à socialização (El-Hussein \& Cronje, 2010, pp. 12-21) e a trabalhar em rede (Martin \& Ertzberger, 2013, pp. 76-85), também podem adaptar a aprendizagem de acordo com o seu gosto ou necessidade para que sirva melhor os seus requisitos (Nedungadi \& Raman, 2012, pp. 659-678). Encontramos também a definição de m-learning agregada ao modo de transmissão, isto é, 3G (Wang \& Shen, 2011, pp. 561-575), 4G (Abachi \& Muhammad, 2014, pp. 491-496), GPRS e wireless (Brown, 2005, pp. 299-315), bluetooth e wi-fi (Puntambekar, Gondal, \& Agrawal, 2009, pp. 1-5), ou à variedade de cursos oferecidos como cursos superiores (Lim, Fadzil, \& Mansor, 2011, pp. 124-137), MBA's (Kukulska-Hulme, Sharples, Milrad, Arnedillo-Sanchez \& Vavoula, 2009, pp. 13-35), aprendizagem ao longo da vida (Trifonova \& Ronchetti, 2003, pp. 1794-1801), MOOC's (Boyatt, Joy, Rocks, \& Sinclair, 2013, pp. 133-145) e outros cursos (Wang, Shen, Novak, \& Pan, 2009, pp. 673-695). O que parece ser um consenso mais alargado é a sua flexibilidade em termos de tempo e local, ou seja, aprender em qualquer lugar e a qualquer hora (Lan \& Sie, 2010, pp. 723-732), a comunicação entre 
intervenientes ser assíncrona, síncrona, espontânea, imediata (Lai, Yang, Chen, Ho \& Chan, 2007, pp. 326-337) e permanente (Huang, Lin, \& Cheng, 2010, pp. 47-58), a vantagem da aprendizagem em rede (Wang, Wu, \& Wang, 2009, pp. 92-118) e a conveniência (Gikas \& Gran, 2013, pp. 18-26). Algumas limitações são mencionadas em termos de adaptabilidade principalmente para os alunos mais velhos que não dominam esta tecnologia (Yanga et al., 2013, pp. 233243), pela dificuldade de apresentar gráficos em pormenor, uma vez que o ecrã é pequeno (El-Hussein \& Cronje, 2010, pp. 12-21), e limitações em hardware principalmente para imprimir e no armazenamento (Hashemi, Azizinezhad, Najafi, \& Nesari, 2011, pp. 2477-2481).

Os autores apresentados, como muitos outros, acreditam que a definição apresentada nos seus artigos é aquela que mais se adapta ao âmbito dos seus trabalhos, contudo, uma tentativa de aproximação para apresentar uma definição genérica parece ter sido pouco explorada.

\section{Metodologia qualitativa}

\section{Base da recolha dos dados}

Nesta investigação, o objetivo é focalizado em encontrar uma forma, a ter em conta pelos interessados, para construírem de uma forma simples, objetiva e rigorosa as definições distance learning, e-learning e m-learning, pelo que, neste contexto, a importância de uma metodologia de pesquisa qualitativa (Creswell \& Miller, 1997) adicionada a uma base empírica (Newbert, 2006) é necessária na construção de características e subcaracterísticas extraídas da revisão da literatura que estejam ligadas diretamente a cada definição.

\section{Análise de dados}

Os 63 artigos analisados, na revisão da literatura para as três definições, permitiram criar um inventário qualitativo, ou seja, foram inseridos no MS Excel todos os dados. A lista resultante dos dados inseridos foi analisada e trabalhada recorrendo inicialmente à técnica de código aberto (Walker \& Myrick, 2006) e posteriormente à técnica de axial code (Kendall, 1999; Simpson \& Tuson, 2003, pp. 25-43) para sintetizar, categorizar e relacionar temas emergentes. Como resultado, seis características surgiram (tecnologia, curso, acessibilidade, adaptabilidade, comunicação e flexibilidade). A etapa seguinte pretendia explorar a quantificação das relações existentes dentro de cada categoria, mas dentro da mesma lista de características obtida inicialmente com o objetivo de contruir 
uma estrutura hierárquica que permita conhecer as subcaracterísticas, ou seja, repetindo as técnicas iniciais obtive doze subcaracterísticas (ver Tabela 1, coluna "subcaracterística").

\section{Resultado qualitativo}

A Tabela 1 pode ser dividida em dois grupos, o primeiro grupo que é composto pelas características principais e subcaracterísticas (coluna 1 e 2 da esquerda para a direita) e o segundo grupo (colunas 3, 4 e 5 da esquerda para a direita) com o resultado das características e subcaracterísticas para distance learning, e-learning, m-learning. $\mathrm{O}$ segundo grupo possui algumas notas de rodapé para as palavras com números. O primeiro grupo deverá ter em conta os seguintes significados:

- tecnologia $=$ conjunto de recursos tecnológicos que disponibilizam ferramentas, processos, procedimentos e que, aplicados a vários propósitos, permitem desenvolver o conhecimento e facilitar o acesso à informação (Gachago, Livingston \& Ivala, 2016, pp. 859-872; Ely, 2008, pp. 244-250). É composto pelas seguintes subcategorias: dispositivos de acesso (aparelho ou mecanismo que suporta a aprendizagem, (Cavus \& Ibrahim, 2009, pp. 78-91)) e modo de transmissão (tecnologia que é empregue para entregar a informação ao aluno (Monahan, McArdle, \& Bertolotto, 2008, pp. 1339-1353);

- curso = é um evento estruturado, com objetivos, com um propósito, com uma metodologia, com conteúdos selecionados sobre determinados temas, com resultados esperados e que após a sua conclusão com sucesso permitem obter uma graduação e o respetivo diploma de reconhecimento (Dally \& Zhang, 1993, pp. 83-91; Pimmel, 2001, pp. 413-421; Stringer \& Lyall, 2012, pp. 48-54);

- acessibilidade $=$ refere-se ao grau de facilidade com que um estudante com deficiência pode aceder e utilizar um sistema de aprendizagem, isto é, plataformas (Park S. Y., 2009, pp. 150-162), ferramentas e conteúdos (Seale \& Cooper, 2010, pp. 1107-1116);

- adaptabilidade $=$ é definido como um processo de aprendizagem personalizado em que um sistema interage com o aluno de acordo com os seus requisitos e preferências (Brusilovsky, 2012, pp. 46-48);

- comunicação = é um processo que consiste na transmissão de uma mensagem ou informação do emissor para o recetor em que este a in- 
terpreta, relaciona-se com o emissor, troca experiências, sentimentos, ideias ou informações (Færch \& Kasper, 1984, pp. 45-63). A forma de comunicação pode ser síncrona (real time, live) ou assíncrona (gravada em áudio e/ou vídeo) (Williamson, Bernhard \& Chamberlin, 2000, pp. 53-61). O tipo de comunicação pode ser interativa (relacionamento de permissão para a pessoa exercer influência sobre o conteúdo ou a forma da comunicação) ou sem qualquer interação (Saka, Southerland, Kittleson, \& Hutner, 2013, pp. 1221-1244) e espontânea, isto é, ad-hoc, não programada, reação natural (Ebner, 2009, pp. 91-100). A ligação da comunicação pode ser permanente, isto é, quando é constante (Wang, $\mathrm{Ci}$ \& Zhan, 2007, pp. 791-795) ou momentânea, ou seja, instantânea ou de curta duração (Sun, Tsai, Finger, Chen, \& Yeh, 2008, pp. 1183-1202). A aprendizagem através da comunicação pode ser Networked, também conhecida por conectivismo, em que a pessoa constrói a sua própria rede e obtém ligações distribuídas por várias comunidades (Goodyear, 2005, pp. 82-101) ou colaborativa, isto é, aprender em conjunto com outros (Gregory, 2015, pp. 63-76);

- flexibilidade = está relacionado com a vantagem de oferecer ao aluno, a escolha de tempo e local para realizar as suas atividades dentro de limites pré-estabelecidos pelo professor (Barak \& Levenberg, 2016, pp. 39-52). É composto pelas subcategorias: local, tempo e flexibilidade do sistema (inclui a flexibilidade de comunicação com os outros intervenientes, flexibilidade da tecnologia e flexibilidade geral da aprendizagem (Alrawi \& Jaber, 2008, pp. 118-128).

A Tabela 1 ilustra de forma clara as características e subcaracterísticas agregadas a cada tipo de entrega de ensino, isto é, distance learning, e-learning e m-learning. Na característica tecnologia, o dispositivo de acesso de distance learning (papel, rádio e TV) é completamente diferente do dispositivo de acesso do e-learning (desktop) e do dispositivo de acesso do m-learning (mobile devices) o que podemos concluir que, nesta característica, a diferença entre o dispositivo de acesso no e-learning é fixa enquanto no m-learning é móvel. No entanto, por exemplo, o laptop é considerado um dispositivo de acesso e-learning quando está ligado à internet com o modo de transmissão por cabo mas pode ser considerado dispositivo de acesso "móvel" quando está ligado à internet utilizando o modo de transmissão WI-FI, $3 \mathrm{G}$ ou 4G. Na subcategoria modo de transmissão, podemos verificar que, a partir do momento da introdução das TIC e das tecnologias de internet, apareceram os dois modos de entrega e-learning e m-learning, pelo que o distance learning possui apenas em termos tecnológicos a TV, o rádio e os leitores de tapes vídeo/áudio; contudo, não devemos confundir a tecnologia TV (simples aparelho de televisão) utilizada no distance learning 
TABELA 1 - CARACTERÍSTICAS E SUBCARACTERÍSTICAS DE DISTANCE LEARNING, E-LEARNING E M-LEARNING

\begin{tabular}{|c|c|c|c|c|}
\hline Característica & Subcaracterística & Distance learning & E-learning & M-learning \\
\hline \multirow[t]{2}{*}{ Tecnologia } & Dispositivos de acesso & Papel, rádio e TV & $\begin{array}{l}\text { Computador de } \\
\text { secretária }\end{array}$ & $\begin{array}{l}\text { iPod, iPhone, iPad, } \\
\text { PDA, laptops }{ }^{1}, \text { MP3 } \\
\text { e tablets (ou seja, } \\
\text { mobile devices }^{2} \text { ) }\end{array}$ \\
\hline & Modo de transmissão & $\begin{array}{l}\text { Correio postal, fax, } \\
\text { televisão, fitas de } \\
\text { vídeo/áudio } \\
\end{array}$ & \begin{tabular}{|l|} 
internet ou \\
intranet (por \\
cabo)
\end{tabular} & \begin{tabular}{|l|} 
GPRS, GSM, \\
Wireless, WI-FI, \\
Bluetooth, $3 G, 4 G$ \\
\end{tabular} \\
\hline Curso & Tipo & $\begin{array}{l}\text { Cursos superiores e } \\
\text { profissionais }\end{array}$ & \begin{tabular}{|l|} 
Curso superior, \\
MBA's, ALV, \\
MOOC, outros \\
\end{tabular} & \begin{tabular}{|l|} 
Curso superior, \\
MBA's, ALV, \\
MOOC $^{3}$, outros \\
\end{tabular} \\
\hline Acessibilidade & $\begin{array}{l}\text { Plataforma tecnológica, } \\
\text { ferramentas e conteúdos }\end{array}$ & Não existe & Existe & Necessita de ajustes \\
\hline Adaptabilidade & Aprendizagem & Não existe & Existe & $\begin{array}{l}\text { Necessita de } \\
\text { ajustes }^{4}\end{array}$ \\
\hline Comunicação & \begin{tabular}{|l} 
- Forma \\
- Tipo \\
- Ligação \\
- Aprendizagem \\
\end{tabular} & $\begin{array}{l}\text {-Assíncrono } \\
\text {-Sem interação } \\
\text {-Não existe } \\
\text {-Isolado } \\
\end{array}$ & $\begin{array}{l}\text {-Asynchronous } \\
\text { e Synchronous } \\
\text {-Interativo } \\
\text {-Momentâneo } \\
\text { - Colaborativa } \\
\end{array}$ & $\begin{array}{l}\text {-Assíncrono e } \\
\text { síncrono } \\
\text {-Espontâneo } \\
\text {-Permanente } \\
\text { - Networked } \\
\end{array}$ \\
\hline Flexibilidade & \begin{tabular}{|l} 
- Local \\
- Tempo \\
- Sistema (tecnologia \\
e comunicação com os \\
intervenientes)
\end{tabular} & \begin{tabular}{|l}
-Estático \\
-Qualquer \\
-Não existe
\end{tabular} & $\begin{array}{l}\text {-Estático } \\
\text {-Qualquer } \\
\text {-Avançada/ } \\
\text { Avançada }\end{array}$ & \begin{tabular}{|l|}
-Dinâmico \\
-Qualquer \\
-Média $/$ Avançada
\end{tabular} \\
\hline
\end{tabular}

FONTE: elaborado pelo autor.

${ }^{1}$ Desde que ligados à rede GPRS, GSM, Wireless, WI-FI, Bluetooth, 3G, 4G.

${ }^{2}$ Possui ainda limitações ao nível de hardware, ou seja, capacidade da bateria, limitações no armazenamento.

${ }^{3}$ Nem todas as plataformas de aprendizagem MOOC possibilitam a visualização em aplicativos móveis para todos os sistemas operativos.

${ }^{4}$ Os alunos mais velhos e que não dominam a tecnologia móvel possuem algumas dificuldades.

${ }^{5} \mathrm{O}$ modo assíncrono é o mais utilizado. O modo síncrono é limitado à disponibilidade dos outros intervenientes.

${ }^{6}$ Devido às limitações que ainda possui como impressão e armazenamento.

com a tecnologia do iTV (interactive TV), que nasceu da junção entre a televisão digital, como plataforma organizativa, e a interatividade como modelo de comunicação (Kim \& Sawhney, 2002, pp. 217-233) e que é classificada de t-learning (Colace, Santo \& Ritrovato, 2008, pp. 1-6). Na característica curso, a grande diferença encontrada entre distance learning e as outras formas de entrega (e-learning e m-learning) é que na oferta de cursos no distance learning existe uma menor oferta, mas, entre e-learning e m-learning, verificamos na prática que o tipo de cursos MOOC (assistimos a vários $\operatorname{cursos}^{1}$ ) oferecidos por

1 Os cursos foram assistidos nas plataformas Coursera, edX, Futurlearn, MiríadaX, Iversity, Udacity e ecolearning. 
algumas plataformas de aprendizagem ainda não possuem aplicações móveis ou se as possuem não são compatíveis com todos os sistemas operativos. $\mathrm{Na}$ característica acessibilidade, isto é, para grupos de alunos com deficiência que ascendem à plataforma de aprendizagem, ferramentas de comunicação, suporte e materiais de aprendizagem, podemos constatar que ainda existe uma ligeira diferença, uma vez que no sistema e-learning existe uma implementação mais significativa do que no sistema de m-learning (Kumar \& Owston, 2016, pp. 263-283; Liu, Li \& Carlsson, 2010, pp. 1211-1219). Na adaptabilidade encontramos diferenças, visto que o m-learning necessita igualmente de ajustes nomeadamente para alunos com mais idade, muitas vezes os smartphones e as aplicações móveis desenvolvidas para a aprendizagem não estão pensadas para os alunos que possuem mais dificuldade nesta tecnologia (Yanga et al., 2013, pp. 233-243). Na característica comunicação, podemos verificar na Tabela 1 que no distance learning é totalmente assíncrono, no e-learning o método mais utilizado é o assíncrono, embora possam ocorrer comunicações síncronas com marcação para que todas as partes estejam disponíveis e no m-learning é mais utilizado o método síncrono embora também exista a possibilidade da comunicação assíncrona, nomeadamente utilizando ferramentas como o email ou o blog (Murphy, Rodríguez-Manzanares \& Barbour, 2011, pp. 583-591). No tipo de comunicação, a diferença entre o distance learning e o e-learning está na interação, visto que no distance learning não existe interação (Chickering \& Ehrmann, 1996, pp. 3-7), no m-learning o tipo de comunicação é espontâneo ((Lee \& Chan, 2007, pp. 201-218). Na aprendizagem com base na comunicação, as diferenças são claras, uma vez que no distance learning a aprendizagem é isolada, mas, com o aparecimento da tecnologia, no e-learning a aprendizagem passou a ser colaborativa e no m-learning a aprendizagem é classificada de "aprendizagem em rede", já que, através do dispositivo móvel, os alunos e os professores facilmente, em qualquer lugar e imediatamente, comunicam com a sua rede ou comunidade (Martin \& Ertzberger, 2013, pp. 76-85). Na característica flexibilidade, podemos verificar que em termos de tempo (subcategoria) não existe qualquer diferença; contudo, o local para distance learning e $e$-learning é estático, uma vez que o aluno não pode estar em movimento, o movimento só pode acontecer no m-learning porque utiliza mobile devices (Popović, Marković, \& Popović, 2016, pp. 412-420).

De acordo com o que foi referido anteriormente, a Figura 1 mostra um esquema gráfico para que de uma forma simples, objetiva, rigorosa e clara se possa identificar as características (caixa com fundo branco) e subcaracterísticas (caixa com fundo azul) a ter em conta quando se pretende construir as definições de distance learning, e-learning e m-learning. 
FIGURA 1 - ESQUEMACOMAS CARACTERÍSTICAS E SUBCARACTERÍSTICAS A TER EM CONTA PARA CONSTRUIR CADA DEFINIÇÃO

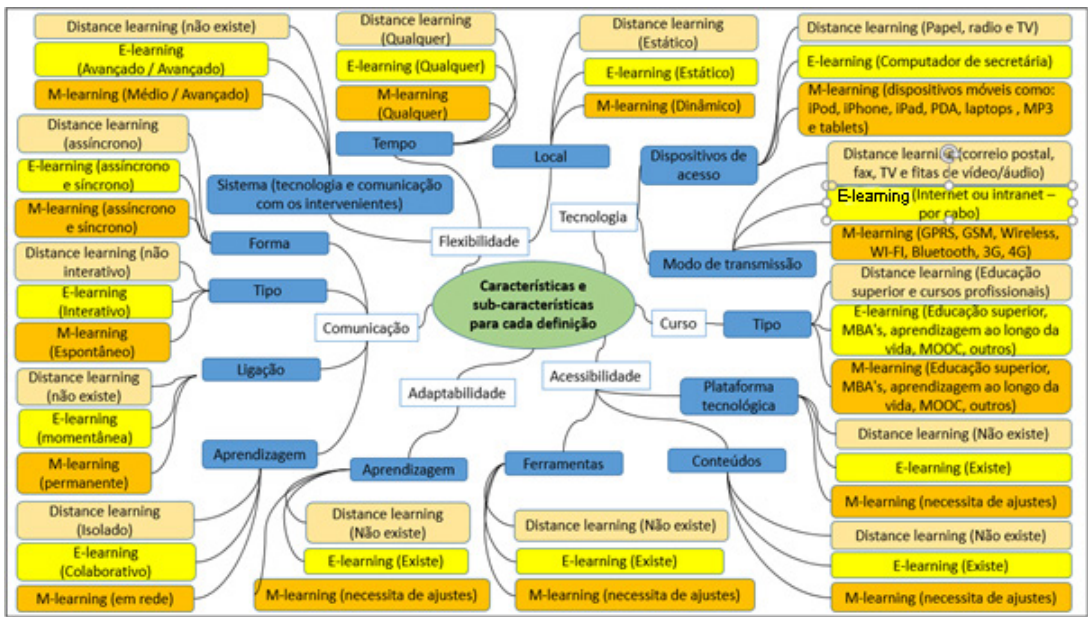

FONTE: elaborado pelo autor.

\section{Limitações}

Questões adicionais sobre este estudo surgem agora, uma vez que não foi possível obter respostas devido à extensão adicional que seria necessário neste trabalho. Para estudos futuros, deve ser explorado uma validação prática, isto é, com base nas características e subcaracterísticas encontradas deve exigir-se definições concretas e avaliá-las junto de um conjunto de espertos nesta área.

\section{Conclusão}

Com base no método escolhido, foi encontrado uma forma para no futuro se construir uma definição com mais rigor e objetividade de distance learning, e-learning e m-learning. A descoberta das características e das subcaracterísticas agregadas a cada definição facilita a construção de definições, visto que existe uma ligação direta entre o conceito final e o seu conteúdo (características 
e subcaracterísticas), para além disso, a partir de agora já temos uma base. É fundamental a utilização conjunta das características e subcaracterísticas na construção de cada definição para que esta seja precisa e rigorosa; isto é, utilizar apenas as características sem subcaracterísticas, ou somente algumas caraterísticas, ou todas as características e algumas subcaracterísticas origina a que a construção da definição não fique totalmente clarificada, ordenada e bem definida e iremos repetir o mesmo erro que constatamos na revisão da literatura (diferentes contextos, definições associadas a interesses e incompletas). Uma definição correta permite atribuir um significado aplicado dentro de um conjunto específico de circunstâncias. Uma definição correta tem que ser específica, concreta e útil para as pessoas que estão envolvidas.

\section{REFERÊNCIAS}

Abachi, H. R. \& Muhammad, G. (2014). The impact of m-learning technology on students and educators. Computers in Human Behavior, 30, 491-496.

Allen, M., Mabry, E., Mattrey, M., Bourhis, J., Titsworth, S. \& Burrell, N. (2004). Evaluating the Effectiveness of Distance Learning: A Comparison Using Meta-Analysis. Journal of Communication, 54(3), 402-420.

Alrawi, K. \& Jaber, K. H. (2008). How flexible is e-learning in the educational institutions: the Arab universities' case study. International Journal of Trade and Global Markets, $1(2), 118-128$.

Alshammari, M., Anane, R. \& Hendley, R. J. (2014). Adaptivity in E-Learning Systems. Eighth International Conference on Complex, Intelligent and Software Intensive Systems (pp. 79-85). Birmingham: IEEE.

Anohina, A. (2005). Analysis of the terminology used in the field of virtual learning. Educational Technology \& Society, 8(3), 91-102.

Banas, E. J. \& Emory, W. F. (1998). History and issues of distance learning. Public Administration Quarterly, 22(3), 365-383.

Barak, M. \& Levenberg, A. (2016). Flexible thinking in learning: An individual differences measure for learning in technology-enhanced environments. Computers \& Education, 99, 39-52.

Bottino, R. M. (2004). The evolution of ICT-based learning environments: which perspectives for the school of the future? British Journal of Educational Technology, 35(5), 553-567. 
Boulos, M. N., Taylor, A. D. \& Breton, A. (2005). A Synchronous Communication Experiment within an Online Distance Learning Program: A Case Study. Telemedicine and e-Health, 11(5), 583-593.

Boyatt, R., Joy, M., Rocks, C. \& Sinclair, J. (2013). What (Use) is a MOOC? International Workshop on Learning Technology for Education in Cloud (pp. 133-145). Kaohsiung: Springer Proceedings.

Brito, P. Q. (2012). Tweens' characterization of digital technologies. Computers \& Education, 59(2), 580-593.

Brown, T. H. (2005). Towards a Model for m-Learning in Africa. International Journal on e-Learning, 4(3), 299-315.

Brusilovsky, P. (2012). Adaptive Hypermedia for Education and Training. In A. M. Paula J. Durlach, Adaptive Technologies for Training and Education. (pp. 46-48). Cambridge: Cambridge University Press.

Cavus, N. \& Ibrahim, D. (2009). m-Learning: An experiment in using SMS to support learning new English language words. British Journal of Educational Technology, 40(1), 78-91.

Chickering, A. W. \& Ehrmann, S. C. (1996). Implementing the Seven Principles: Technology as Lever. AAHE Bulletin, 49(2), 3-7.

Ciampa, K. (2013). Learning in a mobile age: An investigation of student motivation. Journal of Computer Assisted Learning, 30(1), 82-96.

Colace, F., Santo, M. D. \& Ritrovato, P. (2008). From E-Learning to T-Learning. 3rd International Conference on Information and Communication Technologies: From Theory to Applications (pp. 1-6). Damascus: IEEE.

Creswell, J. W. \& Miller, G. A. (1997). Research Methodologies and the Doctoral Process. New Directions for Higher Education, 1997(99), 33-46.

Cropley, A. J. \& Kahl, T. N. (1983). Distance education and distance learning: some psychological considerations. Distance Education, 4(1), 27-39.

Dally, J. W. \& Zhang, G. M. (1993). A Freshman Engineering Design Course. Journal of Engineering Education, 82(2), 83-91.

Dede, C. J. (1990). The Evolution of Distance Learning. Journal of Research on Computing in Education, 22(3), 247-264.

Ding, X., Niu, J. \& Han, Y. (2010). Research on distance education development in China. British Journal of Educational Technology, 41(4), 582-592.

Du, C., Liu, H. \& Wei, L. (2010). On the Characteristics and Application of Integrated E-Learning. 2nd International Conference on e-Business and Information System Security (EBISS) (pp. 659-661). Wuhan: IEEE.

Ebner, M. (2009). Introducing live microblogging: how single presentations can be enhanced by the mass. Journal of research in innovative teaching, 2(1), 91-100. 
El-Hussein, M. O. \& Cronje, J. C. (2010). Defining Mobile Learning in the Higher Education Landscape. Journal of Educational Technology \& Society, 13(3), 12-21.

Ely, D. (2008). Frameworks of educational technology. British Journal of Educational Technology, 39(2), 244-250.

Evans, C. (2008). The effectiveness of m-learning in the form of podcast revision lectures in higher education. Computers \& Education, 50(2), 491-498.

Færch, C. \& Kasper, G. (1984). Two ways of defining communication strategies. Language Learning, 34(1), 45-63.

Gachago, D., Livingston, C. \& Ivala, E. (2016). Podcasts: A technology for all? British Journal of Educational Technology, 47(5), 859-872.

Galusha, J. M. (1997). Barriers to Learning in Distance Education. Interpersonal Computing and Technology Journal, 5(3), 6-14.

Gikas, J. \& Gran, M. M. (2013). Mobile computing devices in higher education: Student perspectives on learning with cellphones, smartphones \& social media. The Internet and Higher Education, 19, 18-26.

Goodyear, P. (2005). Educational Design and Networked Learning: Patterns, Pattern Languages and Design Practice. Australasian Journal of Educational Technology, 21(1), 82-101.

Gregory, S. (2015). Discussion boards as collaborative learning tools. International Journal of Continuing Engineering Education and Life Long Learning, 25(1), 63-76.

Grossnickle, E. M. (2016). Disentangling Curiosity: Dimensionality, Definitions, and Distinctions from Interest in Educational Contexts. Educational Psychology Review, 28(1), 23-60.

Guri-Rosenblit, S. (2005). Distance education' and 'e-learning': Not the same thing. Higher Education, 49(4), 467-493.

Hartshorne, R. \& Ajjan, H. (2009). Examining student decision to adopt web 2.0 technologies: theory and empirical tests. Journal of Computing in Higher Education, 21(3), 183-198.

Hashemi, M., Azizinezhad, M., Najafi, V. \& Nesari, A. J. (2011). What is Mobile Learning? Challenges and Capabilities. Procedia - Social and Behavioral Sciences, 30, 2477-2481.

Heilesen, S. B. (2010). What is the academic efficacy of podcasting? Computers \& Education, 55(3), 1063-1068.

Huang, Y.-M., Lin, Y.-T. \& Cheng, S.-C. (2010). Effectiveness of a Mobile Plant Learning System in a science curriculum in Taiwanese elementary education. Computers \& Education, 54(1), 47-58. 
Hürsen, Ç., Özçınar, Z., Özdamlı, F. \& Uzunboylu, H. (2011). The communicative competences of students and teachers in different levels of education in North Cyprus. Asia Pacific Education Review, 12(1), 59-66.

Isaiah, T. A. \& Martin, C. (2015). Using management procedure gaps to enhance e-learning implementation in Africa. Computers \& Education, 90, 64-79.

Joksimović, S., Gašević, D., Loughin, T. M., Kovanović, V. \& Hatala, M. (2015). Learning at distance: Effects of interaction traces on academic achievement. Computers \& Education, 87, 204-217.

Jones, N., \& Lau, A. (2009). E-learning - a change agent for education? Journal of Applied Research in Higher Education, 1(1), 40-48.

Katz, Y. J. (2002). Attitudes affecting college students' preferences for distance learning. Journal of Computer Assisted Learning, 18(1), 2-9.

Keegan, D. (1996). Foundations of Distance Education. London: Routledge. Studies in Distance Education. Third Edition.

Kendall, J. (1999). Axial Coding and the Grounded Theory Controversy. Western Journal of Nursing Research, 21(6), 743-757.

Kerr, B. (2014). Challenges in global e-learning: What does the literature tell us. 2014 International Conference on Interactive Collaborative Learning (pp. 156-160). Dubai: IEEE.

Kim, C.-J. \& Santiago, R. (2005). Construction of E-learning environments in Korea. Educational Technology Research and Development, 53(4), 108-114.

Kim, P. \& Sawhney, H. (2002). A machine-like new medium - theoretical examination of interactive TV. Media Culture Society, 24(2), 217-233.

King, F. B., Young, M. F., Drivere-Richmond, K. \& Schrader, P. G. (2001). Defining distance learning and distance education. $A A C E, 9(1), 1-14$.

Koper, R. (2005). An Introduction to Learning Design. In: Rob Koper, C. T. Learning Design. (pp. 3-20). Berlin: Springer Berlin Heidelberg.

Korucu, A. T. \& Alkan, A. (2011). Differences between m-learning (mobile learning) and e-learning, basic terminology and usage of m-learning in education. Procedia - Social and Behavioral Sciences, 15, 1925-1930.

Kukulska-Hulme, A., Sharples, M., Milrad, M., Arnedillo-Sanchez, I. \& Vavoula, G. (2009). Innovation in Mobile Learning: A European Perspective. International Journal of Mobile and Blended Learning, 1(1), 13-35.

Kumar, K. L. \& Owston, R. (2016). Evaluating e-learning accessibility by automated and student-centered methods. Educational Technology Research and Development, 64(2), 263-283. 
Lai, C.-H., Yang, J.-C., Chen, F.-C., Ho, C.-W. \& Chan, T.-W. (2007). Affordances of mobile technologies for experiential learning: the interplay of technology and pedagogical practices. Journal of Computer Assisted Learning, 23(4), 326-337.

Lan, Y.-F. \& Sie, Y.-S. (2010). Using RSS to support mobile learning based on media richness theory. Computers \& Education, 55(2), 723-732.

Lee, M. J. \& Chan, A. (2007). Pervasive, lifestyle-integrated mobile learning for distance learners: an analysis and unexpected results from a podcasting study. Open Learning: The Journal of Open, Distance and e-Learning, 22(3), 201-218.

Li, J. (2010). Developing accessible e-learning content. International Conference on Information Science and Engineering, 3 (pp. 2017-2020). Hangzhou: IEEE.

Li, Y., Duan, Y., Fu, Z. \& Alford, P. (2012). An empirical study on behavioural intention to reuse e-learning systems in rural China. British Journal of Educational Technology, 43(6), 933-948.

Lim, T., Fadzil, M. \& Mansor, N. (2011). Mobile Learning via SMS at Open University Malaysia: Equitable, Effective, and Sustainable. International Review of Research in Open and Distance Learning, 12(2), 124-137.

Liu, G. (2010). Influences of e-learning to life-long education. International Conference on Computer Science and Information Technology (pp. 218-222). Chengdu: IEEE.

Liu, Y., Li, H. \& Carlsson, C. (2010). Factors driving the adoption of m-learning: An empirical study. Computers \& Education, 55(3), 1211-1219.

Liyanagunawardena, T. R., Lundqvist, K. Ø. \& Williams, S. A. (2015). Who are with us: MOOC learners on a FutureLearn course. British Journal of Educational Technology, 46(3), 557-569.

Lyashenko, M. S. \& Frolova, N. H. (2014). LMS projects: A platform for intergenerational e-learning collaboration. Education and Information Technologies, 19(3), 495-513.

Mapuranga, B. \& Nyenya, T. (2014). Open and Distance Learning Accessibility to Learners with Disabilities. International Journal of Humanities Social Sciences and Education, 1(4), 1-12.

Martin, F. \& Ertzberger, J. (2013). Here and now mobile learning: An experimental study on the use of mobile technology. Computers \& Education, 68, 76-85.

McGill, T. J. \& Klobas, J. E. (2009). A task-technology fit view of learning management system impact. Computers \& Education, 52(2), 496-508.

Milrad, M. (2003). Mobile Learning: Challenges, Perspectives and Reality. In: János, K. N. (Ed.), Mobile Learning. Essays on Philosophy, Psychology and Education. (pp. 151-164). Vienna: Passagen Verlag.

Monahan, T., McArdle, G. \& Bertolotto, M. (2008). Virtual reality for collaborative e-learning. Computers \& Education, 50(4), 1339-1353. 
Motiwalla, L. F. (2007). Mobile learning: A framework and evaluation. Computers \& Education, 49(3), 581-596.

Murphy, E., Rodríguez-Manzanares, M. A. \& Barbour, M. (2011). Asynchronous and synchronous online teaching: Perspectives of Canadian high school distance education teachers. British Journal of Educational Technology, 42(4), 583-591.

Nedungadi, P. \& Raman, R. (2012). A new approach to personalization: integrating e-learning and m-learning. Educational Technology Research and Development, 60(4), 659-678.

Newbert, S. L. (2006). Empirical research on the resource-based view of the firm: an assessment and suggestions for future research. Strategic Management Journal, 28(2), 121-146.

Ng, W. \& Nicholas, H. (2013). A framework for sustainable mobile learning in schools. British Journal of Educational Technology, 44(5), 695-715.

Ozkan, S. \& Koseler, R. (2009). Multi-dimensional students' evaluation of e-learning systems in the higher education context: An empirical investigation. Computers \& Education, 53(4), 1285-1296.

Pahl, C. (2013). Managing evolution and change in web-based teaching and learning environments. Computers \& Education, 40(2), 99-114.

Park, S. Y. (2009). An Analysis of the Technology Acceptance Model in Understanding University Students' Behavioral Intention to Use e-Learning. Journal of Educational Technology \& Society, 12(3), 150-162.

Park, Y. (2011). A pedagogical framework for mobile learning: Categorizing educational applications of mobile technologies into four types. International Review of Research in Open and Distance Learning, 12(2), 78-102.

Passerini, K. \& Granger, M. J. (2000). A developmental model for distance learning using the Internet. Computers \& Education, 34(1), 1-15.

Peters, K. (2007). m-Learning: Positioning educators for a mobile, connected future. IRRODL, 8(2), 1-16.

Pimmel, R. (2001). Cooperative Learning Instructional Activities in a Capstone Design Course. Journal of Engineering Education, 90(3), 413-421.

Popović, O., Marković, D. S. \& Popović, R. (2016). mTester-Mobile learning system. Computer Applications in Engineering Education, 24(3), 412-420.

Puntambekar, D., Gondal, S. \& Agrawal, M. (2009). Province of multiuser m-learning environment using artificial intelligence \& bluetooth techniques. First Asian Himalayas International Conference on Internet (pp. 1-5). Kathmundu,: IEEE.

Raaij, E. M. \& Schepers, J. J. (2008). The acceptance and use of a virtual learning environment in China. Computers \& Education, 50(3), 838-852. 
Ramírez, M., Rogan, J., Valdivia, J. A., Varas, A. \& Kiwi, M. (2016). Diversity Characterization of Binary Clusters by Means of a Generalized Distance. Zeitschrift für Physikalische Chemie, 230(5-7), 977-989.

Rauner, F. \& Maclean, R. (2008). Handbook of Technical and Vocational Education and Training Research. Rotterdam: Springer Netherlands.

Rosenberg, M. J. (2002). E-Learning: Strategies for Delivering Knowledge in the Digital Age. New York: McGraw-Hill.

Saba, F. (2016). Theories of Distance Education: Why They Matter. New Directions for Higher Education, 2016, 21-30.

Saka, Y., Southerland, S. A., Kittleson, J. \& Hutner, T. (2013). Understanding the Induction of a Science Teacher: The Interaction of Identity and Context. Research in Science Education, 43(3), 1221-1244.

Sarrab, M., Shibli, I. A., \& Badursha, N. (2016). An Empirical Study of Factors Driving the Adoption of Mobile Learning in Omani Higher Education. IRRODL, 17(4), 331-349.

Schweizer, H. (2004). E-Learning in Business. Journal of Management Eduction, Volume 28(6), 674-692.

Seale, J. \& Cooper, M. (2010). E-learning and accessibility: An exploration of the potential role of generic pedagogical tools. Computers \& Education, 54(4), 1107-1116.

Selim, H. M. (2007). Critical success factors for e-learning acceptance: Confirmatory factor models. Computers \& Education, 49(2), 396-413.

Simpson, M. \& Tuson, J. (2003). Using Observations in Small-Scale Research: A Beginner's Guide. Revised Edition. Using Research. Edinburgh: ERIC.

Stringer, M. D. \& Lyall, P. (2012). Design, implementation, and evaluation of a postgraduate diploma in Surgical Anatomy. Anatomical Sciences Education, 5(1), 48-54.

Sun, P.-C., Tsai, R. J., Finger, G., Chen, Y.-Y. \& Yeh, D. (2008). What drives a successful e-Learning? An empirical investigation of the critical factors influencing learner satisfaction. Computers \& Education, 50(4), 1183-1202.

Tabuencaa, B., Kalzb, M., Drachslerb, H. \& Specht, M. (2015). Time will tell: The role of mobile learning analytics in self-regulated learning. Computers \& Education, 89, 53-74.

Tarus, J. K., Gichoya, D. \& Muumbo, A. (2015). Challenges of Implementing E-Learning in Kenya: A Case of Kenyan Public Universities. International Review of Research in Open and Distance Learning, 16(1), 120-140.

Trifonova, A. \& Ronchetti, M. (2003). Where is Mobile Learning Going? World Conference on E-Learning in Corporate, Government, Healthcare, and Higher Education (pp. 1794-1801). Chesapeake: AACE. 
Tuul, S., Banzragch, O. \& Saizmaa, T. (2016). E-learning in Mongolian Higher Education. International Review of Research in Open and Distance Learning, 17(2), 181-193.

Uzunboylu, H., \& Ozdamli, F. (2011). Teacher perception for m-learning: scale development and teachers' perceptions. Journal of Computer Assisted Learning, 27(6), 544-556.

VanDerLinden, K. (2014). Blended Learning as Transformational Institutional Learning. New Directions for Higher Education, 2014(165), 75-85.

Wachter, B. (2004). The Bologna Process: developments and prospects. European Journal of Education, 39(3), 265-273.

Wang, M. \& Shen, R. (2011). Message design for mobile learning: Learning theories, human cognition and design principles. British Journal of Educational Technology, 43(4), 561-575.

Wang, M., Ci, L. \& Zhan, P. (2007). Applying Wireless Sensor Networks to ContextAwareness in Ubiquitous Learning. International Conference on Natural Computation (pp. 791-795). Haikou: IEEE.

Wang, M., Shen, R., Novak, D. \& Pan, X. (2009). The impact of mobile learning on students' learning behaviours and performance: Report from a large blended classroom. British Journal of Educational Technology, 40(4), 673-695.

Wang, Y.-S., Wu, M.-C. \& Wang, H.-Y. (2009). Investigating the determinants and age and gender differences in the acceptance of mobile learning. 40(1), 92-118.

Warburton, S. (2009). Second Life in higher education: Assessing the potential for and the barriers to deploying virtual worlds in learning and teaching. British Journal of Educational Technology, 40(3), 414-426.

Williamson, C., Bernhard, J. T. \& Chamberlin, K. (2000). Perspectives on an internetbased synchronous distance learning experience. Journal of Engineering Education, 89(1), 53-61.

Woodfine, B., Nunes, M. B. \& Wright, D. (2008). Text-based synchronous e-learning and dyslexia: Not necessarily the perfect match! Computers \& Education, 50(3), 703-717.

Yanga, G., Chenb, N.-S., Kinshukc, Sutinena, E., Andersond, T. \& Wenc, D. (2013). The effectiveness of automatic text summarization in mobile learning contexts. Computers \& Education, 68, 233-243.

Texto recebido em 07 de setembro de 2017. Texto aprovado em 03 de dezembro de 2017. 
